\title{
Evaluation of Intraobserver and Interobserver Reliability of Mechanical Axis Alignment Measure of the Lower Limb through the Panoramic Radiograph in Patients in the Preoperative and Postoperative Periods of Total Knee Arthroplasty
}

\author{
Murilo Barroso Matos ${ }^{1}$, José Leonardo Rocha de Faria ${ }^{2 *}$, Douglas Mello Pavão², Márcio Liu Sandt ${ }^{3}$, \\ Breno Chaves de Almeida Pigozzo², Pedro Guilme Teixeira de Sousa Filho", \\ Rodrigo Pires e Albuquerque ${ }^{2}$
}

${ }^{1}$ CLIFOR-Orthopedics and Fractures Clinic, Vitória, Brazil

${ }^{2}$ Knee Surgery Center, National Institute of Traumatology and Orthopedics, Rio de Janeiro, Brazil

${ }^{3}$ Genutarso-Orthopedics Clinic, Rio de Janeiro, Brazil

${ }^{4}$ Genu Surgery Group, Fortaleza, Brazil

Email: *drjoseleonardorocha@gmail.com

How to cite this paper: Matos, M.B., de Faria, J.L.R., Pavão, D.M., Sandt, M.L., de Almeida Pigozzo, B.C., de Sousa Filho, P.G.T. and e Albuquerque, R.P. (2020) Evaluation of Intraobserver and Interobserver Reliability of Mechanical Axis Alignment Measure of the Lower Limb through the Panoramic Radiograph in Patients in the Preoperative and Postoperative Periods of Total Knee Arthroplasty. Open Journal of Orthopedics, 10, 221-233.

https://doi.org/10.4236/ojo.2020.109024

Received: April 18, 2020

Accepted: September 15, 2020

Published: September 18, 2020

Copyright $\odot 2020$ by author(s) and Scientific Research Publishing Inc. This work is licensed under the Creative Commons Attribution International License (CC BY 4.0).

http://creativecommons.org/licenses/by/4.0/

\begin{abstract}
Objective: To evaluate intraobserver and interobserver reliability of the measurement of the mechanical axis of the lower limb by means of the panoramic radiograph of lower limbs by physicians with different levels of medical practice. Method: A retrospective cross-sectional study with preoperative and postoperative radiographic analysis of a group of 100 patients submitted to total knee arthroplasty. Results: It was observed that there is a highly significant intraobserver and interobserver agreement $(\mathrm{p}<0.0001)$, both preoperatively and postoperatively, among four observers with different training levels. Conclusion: The panoramic radiograph of the lower limbs is a reliable method for measuring the mechanical axis of the lower limb in patients submitted to total knee arthroplasty, regardless of the level of medical practice of the evaluator.
\end{abstract}

\section{Keywords}

Knee, Mechanical Axis, Total Knee Arthroplasty

\section{Introduction}

The mechanical axis of the lower limbs (Figure 1) is traditionally defined by an 
imaginary line drawn from the center of the femoral head to the center of the ankle joint. We consider a mechanical axis to be neutral when this line crosses the center of the knee joint, which is usually immediately medial to the tibial spine. If this line is found medially to the center of the knee joint, we define the mechanical axis as varus, while on the valgus mechanical axis this line is located laterally to the center of the knee [1].

The alignment of the lower limb is an important indicator of load distribution across the knee. Abnormalities in the mechanical axis of the lower limb are considered important factors in the progression of knee osteoarthritis [2].

Knee osteoarthritis is known to be characterized by a functional and structural failure of synovial tissue, with erosion and loss of articular cartilage and subchondral bone, in addition to degenerative meniscal changes. It usually results in progressive knee deformity, with deviation of the mechanical axis of the lower limb in varus or valgus [3].

Studies associate varus mechanical axis deviation with greater progression of arthritis in the medial compartment of the knee, while valgus axis deviation is associated with arthritis progression in the lateral compartment, the greater severity of the axis deviation, the greater is the degree of functional loss. Surgeries to correct limb alignment, such as total knee arthroplasty and tibial osteotomy work by reducing deformity and the limitation caused by osteoarthritis. In order to perform these procedures correctly, proper preoperative planning is essential, through the measurement of altered anatomical parameters such as the mechanical axis of the lower limb [4] [5].

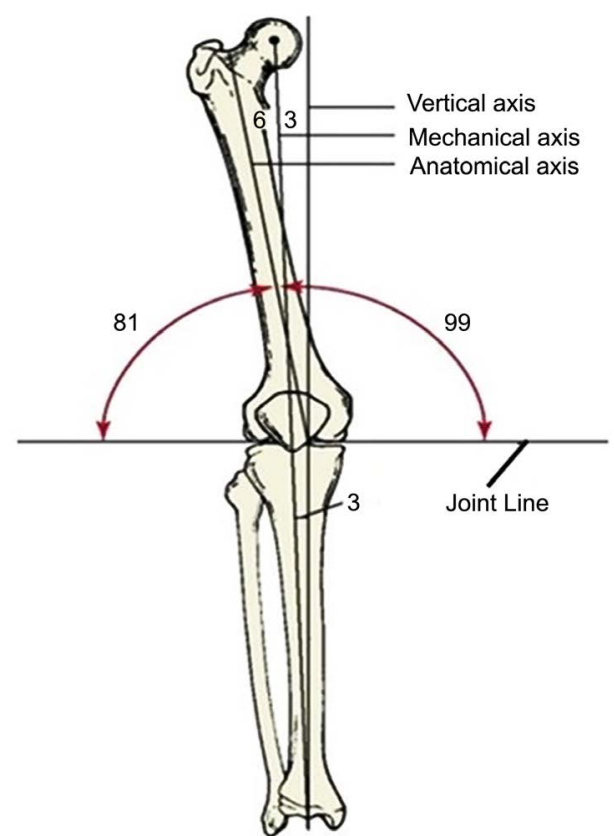

Figure 1. The mechanical axis of the lower limb is defined by a line drawn from the center of the femoral head to the center of the ankle joint. We consider a mechanical axis to be neutral when this line runs through the center of the knee joint, which is usually immediately medial to the tibial spine. 
Total knee arthroplasty (TKA) is a highly complex orthopedic procedure and is indicated for the treatment of patients with severe knee osteoarthritis. Its objective is pain relief, improvement of joint function and correction of knee deformities. The restoration of mechanical knee alignment is one of the main factors involved in the success of the surgical procedure and long-term survival of the implants [6].

Panoramic radiography of the lower limbs is considered the gold standard test for determining the mechanical axis of the lower limb, and the center of the femoral head, knee and ankle can be seen in the same image. This method has already demonstrated good reproducibility of the mechanical axis during the support phase of the gait cycle and good correlation when compared to the axis measurements obtained by navigation. Some recent studies have questioned the reliability of this method of evaluating the mechanical axis of the lower limb, depending on the examiner's experience [7] [8].

The aim of the present study is to evaluate the inter-observer and intra-observer reliability of the measurement of the mechanical axis through panoramic radiography of the lower limbs in patients in the pre- and postoperative period of total knee arthroplasty by doctors of different educational levels.

\section{Material and Methods}

The study was submitted for evaluation and approval by the institution's Research Ethics Committee.

The sample consisted of 100 patients who underwent total knee arthroplasty in 2015, chosen at random, and who had quality radiographs of pre- and postoperative lower limbs scanned into the institution's system, regardless of the age or sex of the participants. The " $n$ " in our study is based on previous studies similar to ours. With this criterion, we determined a number of patients similar to publications with the highest number of patients [9] [10] [11] [12]. No patient was submitted to a new examination due to the present study. Patients who had undergone surgical procedures prior to total knee arthroplasty to correct the alignment of the evaluated limb, such as tibial or femoral osteotomy, were excluded.

Panoramic radiography of the lower limbs is performed with a patient in orthostasis, bipedal load, feet together and parallel, $90^{\circ}$ radius centered at the knee and collimation including hip and ankle. The radiography device used was the Shimatzo, with a $50 \mathrm{KV}$ and $40 \mathrm{~mA}$ technique. All radiographs taken are in digital format.

On the radiographs, the mechanical axis of the lower limb subjected to the surgical procedure was evaluated, being considered by a line from the center of the femoral head to the center of the knee and a second line following from the center of the knee to the center of the talus.

The measurement of the mechanical axis was performed in a computer program used by the institution (mDicom Viewer) (Figure 2). The lines of the mechanical axis of the lower limb were drawn manually by the evaluator using the 


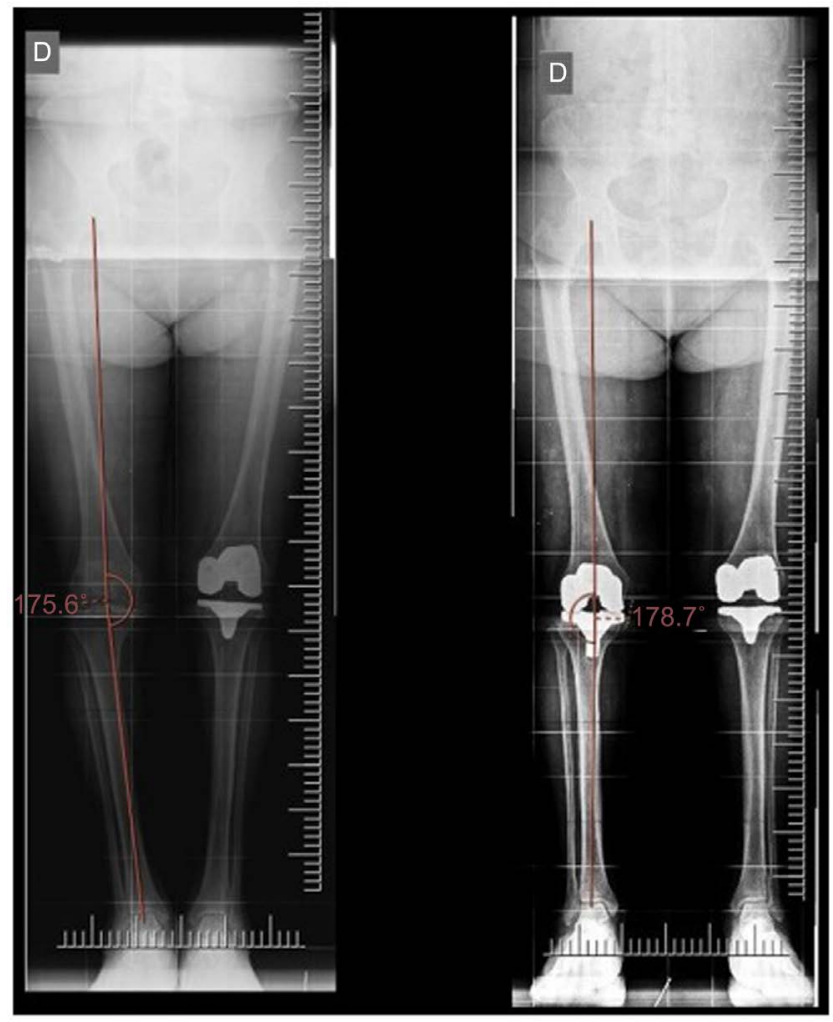

Figure 2. Method of measuring the mechanical axis of the lower limb on panoramic radiography using a computer program used at the Institute.

program's own tool. From the drawn lines, the program indicates the angulation of the measured mechanical axis. The angle of $0^{\circ}$ was defined as a neutral mechanical axis, [13] while negative angles were defined as varus axes, and positive angles were defined as valgus axes.

Each examiner evaluated a preoperative radiograph and a postoperative radiograph of the 100 patients that comprised the sample, at two different times. The second evaluation of the same radiographs occurred 3 months after the first evaluation. The selected evaluators were a second-year resident in Orthopedics and Traumatology, a student in the knee surgery training course, a knee surgeon with less than 10 years of training and a knee surgeon with more than 10 years of training. The evaluators were blinded, having no access to any data that could identify the patients examined.

\section{Statistical Analysis}

The descriptive analysis presented the observed data in tables, expressed by the mean, median, interquartile range $(\mathrm{Q} 1-\mathrm{Q} 3)$, minimum and maximum for the measurement of the mechanical axis. The intra- and inter-observer agreement of the measurement of the mechanical axis was analyzed by the intraclass correlation coefficient (ICC) of a mixed two-way model and absolute agreement. The criterion for determining significance was set at $1 \%$. The statistical analysis was processed using the statistical software SPSS version 20.0. 


\section{Results}

The sample consisted of $51 \%$ patients undergoing TKA on the left knee, and $49 \%$ undergoing TKA on the right knee. 67 patients were female and 33 male. The mean age of the patients was 69.77 years old ( $\min 35$ - max 94). The average value for the preoperative mechanical axis in the first evaluation of the sample was $(-7.48)$ for the 2 nd year resident of Orthopedics and Traumatology, $(-8.39)$ for the student in knee surgery training course, $(-6.58)$ for the knee surgeon with more than 10 years of experience and $(-8.69)$ for the knee surgeon with less than 10 years of experience, reflecting a possible predominance of varus mechanical axes in the sample, in agreement with the known epidemiological distribution of the disease. The average value for the postoperative mechanical axis in the first evaluation of the sample was $(-2.17)$ for the 2 nd year resident of Orthopedics and Traumatology, $(-2.08)$ for the student in knee surgery training course, $(-1.96)$ for the knee surgeon with more than 10 years of experience and $(-2.77)$ for the knee surgeon with less than 10 years of experience, reflecting an improvement in the mechanical alignment of the lower limb after TKA, however, some degree of varus deviation of the axis still persists.

The mean value for the preoperative mechanical axis in the second sample evaluation was (-6.61) for the 2nd year resident of Orthopedics and Traumatology, $(-7.71)$ for the student in knee surgery training course, $(-7.53)$ for the knee surgeon with more than 10 years of experience and $(-8.14)$ for the knee surgeon with less than 10 years of experience, while for the postoperative mechanical axis the values were $(-2.20)$ for the 2 nd year resident of Orthopedics and Traumatology, $(-1.72)$ for the student in knee surgery training course, $(-2.36)$ for the knee surgeon with over 10 years of experience and $(-2.85)$ for the knee surgeon with less than 10 years of experience, reproducing the epidemiological distribution found in the first assessment.

Table 1 provides the descriptive (mean, standard deviation, median, interquartile range [Q1 - Q3], minimum and maximum) of the measurement of the mechanical axis according to the observer, pre and postoperative and M1 and M2 evaluation times ( 3 months after), in the total sample $(\mathrm{n}=100)$.

The intra-observer analysis of the measurement of the mechanical axis was evaluated by the intraclass correlation coefficient (ICC) of a mixed two-way model and absolute agreement.

It is known that the closer the ICC is to one (1), the stronger (or perfect) the agreement is, in this case, the measures are similar under the numerical (quantitative) aspect. On the other hand, the closer to zero (0), the greater the disagreement, that is, it means that they do not "reproduce" and the differences observed are not by chance.

According to FLEISS [14], it can be said that:

ICC $<0.40$ - poor agreement

$0.40 \leq$ ICC $<0.75$-satisfactory to good agreement.

ICC $\geq 0.75$ - excellent agreement. 
Table 1. Complete description of the measurement of the mechanical axis in the total sample.

\begin{tabular}{|c|c|c|c|c|c|c|c|c|c|}
\hline Observer & Moment of evaluation & Moment & $\mathbf{n}$ & mean & SD & median & IR & minimum & maximum \\
\hline \multirow{4}{*}{$\begin{array}{l}\text { 2nd year } \\
\text { resident doctor }\end{array}$} & preoperative & M1 & 100 & -7.48 & 14.38 & -9.6 & $-17.0-1.0$ & -45.5 & 36 \\
\hline & preoperative & M2 & 100 & -6.61 & 14.84 & -9.1 & $-17.2-2.7$ & -43.5 & 36.2 \\
\hline & postoperative & M1 & 100 & -2.17 & 4.27 & -2.5 & $-5.3-0.8$ & -14.3 & 9.2 \\
\hline & postoperative & M2 & 100 & -2.20 & 4.38 & -2.4 & $-5.1-0.1$ & -13.4 & 7.6 \\
\hline \multirow{4}{*}{$\begin{array}{l}\text { stage of } \\
\text { improvement in } \\
\text { knee surgery }\end{array}$} & preoperative & M1 & 100 & -8.39 & 14.63 & -10.8 & $-17.0-0.0$ & -45 & 38 \\
\hline & preoperative & M2 & 100 & -7.71 & 14.90 & -10.1 & $-17.3-2.7$ & -45 & 35 \\
\hline & postoperative & M1 & 100 & -2.08 & 3.94 & -2.2 & $-4.9-0.0$ & -14.2 & 7.1 \\
\hline & postoperative & M2 & 100 & -1.72 & 3.95 & -1.6 & $-4.1-0.0$ & -13.6 & 7.9 \\
\hline \multirow{4}{*}{$\begin{array}{l}\text { Surgeon }>10 \text { years } \\
\text { of experience }\end{array}$} & preoperative & M1 & 100 & -6.58 & 14.67 & -9.4 & $-16.9-2.9$ & -41.8 & 34.5 \\
\hline & preoperative & M2 & 100 & -7.53 & 14.26 & -10.4 & $-16.8-1.3$ & -42.1 & 36.9 \\
\hline & postoperative & M1 & 100 & -1.96 & 4.53 & -1.9 & $-4.7-0.3$ & -14.3 & 8.8 \\
\hline & postoperative & M2 & 100 & -2.36 & 4.74 & -3.0 & $-5.6-1.2$ & -14.7 & 10.9 \\
\hline \multirow{4}{*}{$\begin{array}{c}\text { Surgeon }<10 \text { years } \\
\text { of experience }\end{array}$} & preoperative & M1 & 100 & -8.69 & 14.72 & -11.0 & $-18.2--0.1$ & -50.6 & 36.1 \\
\hline & preoperative & M2 & 100 & -8.14 & 14.95 & -10.7 & $-18.1-2.0$ & -50.3 & 36 \\
\hline & postoperative & M1 & 100 & -2.77 & 4.66 & -2.7 & $-6.0-0.4$ & -13.9 & 8 \\
\hline & postoperative & M2 & 100 & -2.85 & 4.51 & -2.7 & $-6.1-0.3$ & -13.5 & 8.3 \\
\hline
\end{tabular}

SD: Standard deviation. IR: interquartile range (Q1 - Q3).

Table 2 provides the intraclass correlation coefficient (ICC), its respective 95\% confidence interval (95\% CI) and descriptive level ( $\mathrm{p}$ value) for the measurements of the mechanical axis of each moment (M1 and M2) according to the level training: 2nd year resident, for the student in knee surgery training course, knee surgeon with more than 10 years of experience and knee surgeon with less than 10 years of experience in the total sample $(n=100)$.

It can also be said that narrow confidence intervals (CI) express greater precision, on the other hand, wide intervals express low precision, that is, less reliable. It is interesting to observe the lower limit of the $95 \% \mathrm{CI}$, as it expresses the lowest expected value for the ICC, that is, the higher the lower limit the better the quality of agreement.

Similarly, the inter-observer analysis of the measurement of the mechanical axis was evaluated by the intraclass correlation coefficient (ICC) of a mixed two-way model and absolute agreement. Table 3 and Table 4 provide the intraclass correlation coefficient (ICC), its respective 95\% confidence interval (95\% $\mathrm{CI}$ ) and the descriptive level ( $\mathrm{p}$ value) for the measurement of the mechanical axis in the pre and postoperative period. each moment (M1 and M2), for the total sample $(\mathrm{n}=100)$.

It was observed that there is a highly significant intra-observer and inter-observer agreement $(\mathrm{p}<0.0001)$ between the two moments (M1 and M2 - 3 months after M1), both preoperatively and postoperatively for the four evaluators. Although highly significant, an inherent order in the degree of agreement is identified, 
Table 2. Analysis of intra observer agreement in the total sample $(n=100)$.

\begin{tabular}{cccc}
\hline Observer & ICC & CI 95\% & p value \\
\hline Preoperative & & & \\
2nd year resident doctor & 0.92 & $0.88-0.94$ & $<0.0001$ \\
stage of improvement in knee surgery & 0.92 & $0.88-0.95$ & $<0.0001$ \\
Surgeon $>$ 10 years of experience & 0.92 & $0.89-0.95$ & $<0.0001$ \\
Surgeon $<10$ years of experience & 0.97 & $0.96-0.98$ & $<0.0001$ \\
Postoperative & & & \\
2nd year residente doctor & 0.83 & $0.75-0.88$ & $<0.0001$ \\
stage of improvement in knee surgery & 0.95 & $0.93-0.97$ & $<0.0001$ \\
Surgeon $>10$ years of experience & 0.86 & $0.79-0.90$ & $<0.0001$ \\
Surgeon $<10$ years of experience & 0.97 & $0.95-0.98$ & $<0.0001$ \\
\hline
\end{tabular}

ICC: Intraclass Correlation Coefficient for absolute agreement. CI 95\%: $95 \%$ confidence interval for the ICC. Note: Mixed two-way model.

Table 3. Pre-operative inter observer concordance analysis of the total sample $(n=100)$.

\begin{tabular}{|c|c|c|c|}
\hline Observer $1 \times$ Observer 2 & ICC & CI $95 \%$ & $\mathrm{p}$ value \\
\hline \multicolumn{4}{|l|}{ Preoperative of $\mathrm{M} 1$} \\
\hline resident doctor $\times$ improvement in knee surgery & 0.98 & $0.96-0.98$ & $<0.0001$ \\
\hline resident doctor $\times$ surg. $>10$ years & 0.92 & $0.88-0.94$ & $<0.0001$ \\
\hline resident doctor $\times$ surg. $<10$ years & 0.94 & $0.91-0.96$ & $<0.0001$ \\
\hline improvement in knee surgery $\times$ surg. $>10$ years & 0.90 & $0.85-0.94$ & $<0.0001$ \\
\hline improvement in knee surgery $\times$ surg. $<10$ years & 0.93 & $0.90-0.96$ & $<0.0001$ \\
\hline$>10$ years $x<10$ years & 0.87 & $0.80-0.91$ & $<0.0001$ \\
\hline \multicolumn{4}{|l|}{ Preoperative of $\mathrm{M} 2$} \\
\hline resident doctor $\times$ improvement in knee surgery & 0.94 & $0.91-0.96$ & $<0.0001$ \\
\hline resident doctor $\times$ surg. $>10$ years & 0.92 & $0.88-0.94$ & $<0.0001$ \\
\hline resident doctor $\times$ surg. $<10$ years & 0.84 & $0.77-0.89$ & $<0.0001$ \\
\hline improvement in knee surgery $\times$ surg. $>10$ years & 0.90 & $0.85-0.93$ & $<0.0001$ \\
\hline improvement in knee surgery $\times$ surg. $<10$ years & 0.83 & $0.76-0.88$ & $<0.0001$ \\
\hline$>10$ years $x<10$ years & 0.90 & $0.86-0.93$ & $<0.0001$ \\
\hline
\end{tabular}

ICC: Intraclass Correlation Coefficient for absolute agreement. CI 95\%: 95\% confidence interval for the ICC. Note: Mixed two-way model.

Table 4. Postoperative inter observer concordance analysis of the total sample $(n=100)$.

\begin{tabular}{cccc}
\hline Observer $1 \times$ Observer 2 & ICC & CI 95\% & p value \\
\hline Postoperative of $\mathrm{M} 1$ & & & \\
\hline resident doctor $\times$ improvement in knee surgery & 0.92 & $0.88-0.94$ & $<0.0001$ \\
resident doctor $\times$ surg. $>10$ years & 0.81 & $0.73-0.87$ & $<0.0001$ \\
resident doctor $\times$ surg. $<10$ years & 0.90 & $0.84-0.93$ & $<0.0001$ \\
\hline
\end{tabular}




\section{Continued}

improvement in knee surgery $\times$ surg. $>10$ years

improvement in knee surgery $\times$ surg. $<10$ years

$>10$ years $\times<10$ years

Postoperative of M2

resident doctor $\times$ improvement in knee surgery

resident doctor $\times$ surg. $>10$ years

resident doctor $\times$ surg. $<10$ years

improvement in knee surgery $\times$ surg. $>10$ years

improvement in knee surgery $\times$ surg. $<10$ years

$>10$ years $x<10$ years
0.82

$0.74-0.87$

$0.81-0.92$

$0.67-0.84$

0.77

0.76

0.85

0.74

0.84

0.77

0.83
$<0.0001$

$<0.0001$

$<0.0001$

$<0.0001$

$<0.0001$

$<0.0001$

$<0.0001$

$<0.0001$

$<0.0001$

ICC: Intraclass Correlation Coefficient for absolute agreement. CI 95\%: 95\% confidence interval for the ICC. Note: Mixed two-way model.

especially in the postoperative period, the best being the surgeon with more than 10 years of experience, followed by the student in knee surgery training course, then the surgeon with less than 10 years of experience and finally the 2nd year resident of Orthopedics and Traumatology.

\section{Discussion}

The importance of the correct clinical and radiographic evaluation in patients with osteoarticular degenerative disease in the lower limbs, especially knee osteoarthritis, has long been debated for a long time, due to its close relationship with image changes such as poor alignment and decreased joint space and progression disease. Numerous works in the literature have been approaching with different approaches, but all have been systematic in the importance between the radiographic correlation and the progression of the disease and, mainly, in obtaining standardized image studies with good reproducibility and confidence [15] [16] [17].

Some studies have established a good relationship between poor alignment of the limb and the progression of osteoarthritis [18]. However, it is still controversial whether poor alignment predicts the incidence of this disease [10]. Our study aims to define the reliability of the method of panoramic radiography of lower limbs. However, we consider it essential to discuss studies that, based on this method, obtained an accurate assessment of patients with osteoarticular disease, thus calling the orthopedic class to rediscuss the importance of this exam and revalidating it as a fundamental exam for assessing such patients.

Sharma et al. found in their study that varus increases the risk of progression to medial osteoarthritis and valgus increases the risk of developing progression of lateral osteoarthritis. In addition, poor alignment is a predictor of significant functional loss and this effect can be seen in a short period of 48 months [2].

Brouwer et al. reported, through a study evaluating panoramic radiographs of the lower limbs, a good correlation between poor alignment of the lower limb 
and progression of knee osteoarthritis [18]. Su Chan Lee et al. questioned the relationship between lateral compartment arthritis and knee valgus axis deviation. As a conclusion, they presented that the varus axis deviation was found paradoxically in one third of patients with advanced lateral arthritis. In addition, they concluded that $\mathrm{x}$-ray images taken in full extension underestimated the degree of severity of osteoarthritis [10].

Hunter et al. described the role of biomechanics and alignment in knee osteoarthritis and the implications for imaging studies. They concluded that poor alignment in varus or valgus increases the risk of medial and lateral osteoarthritis, respectively. Regarding the progression of the disease, they found that in the presence of knee osteoarthritis, poor alignment is associated with an acceleration of structural deterioration in the compartment subjected to abnormal load, with a 4 -fold increase in the progression of medial osteoarthritis in the varus and an increase 2 to 5 times of progression of lateral osteoarthritis in the valgus [10].

Hanna et al. pointed out a significant disagreement between radiological diagnosis of osteoarthritis and knee pain. Some studies emphasize, because of this, the need for other evaluation methods such as Magnetic Resonance Imaging (MRI), a more specific method capable of identifying lesions such as bone marrow edema, subchondral bone defect, synovitis and effusion [16].

Katia Turcot et al. studied not only changes in alignment on the axis, but also their compensations in gait and concluded that such compensations are significantly influenced by axis deviation. Patients with varus knees significantly increased gait displacement in the sagittal and coronal plane compared to patients with valgus knee [7].

These studies call attention to the importance of establishing good quality image standards when performing panoramic radiography of the lower limbs, as well as medical training to evaluate the images and obtain measures of good reliability. Our study resumes the discussion around the importance of panoramic radiographic evaluation of the lower limbs, as it confirms the reliability and potential of the method, in a study involving a Brazilian population.

Skytta et al. assessed the reliability of panoramic radiography of lower limbs in determining alignment of lower limbs before and after total knee arthroplasty (TKA) in a population in Finland. The authors found an excellent correlation between the mechanical axis, the tibiofemoral angle and the alignment of the femoral and tibial implants on two consecutive radiographs after TKA. There was also an excellent interobserver and intraobserver correlation, facts that corroborate the data found in our study [17].

Hinman et al. demonstrated a good correlation between the mechanical and anatomical axis in a patient with osteoarthritis of the medial knee using the panoramic radiography of the lower limbs for both measurements. Our study focused on comparing the measurements of the mechanical axis at different times, as we believe it is sufficient for validation of the method and comparison between observers. However, we agree that the evaluation of the anatomical axis through panoramic radiography is also essential for preoperative evaluation and 
planning [19].

Kraus et al. evaluated the axis measurements on panoramic radiographs and in Postero-anterior incidence with fixed knee flexion to determine knee alignment both mechanical and anatomical, in patients with osteoarthritis. The authors reached a compensatory angle value for the mechanical axis from the anatomical axis [20]. Issa et al. used panoramic radiographs of the lower limb with knee in semi-flexion to obtain alignment measurements. In addition, they established correlations between alignment and MRI findings [21].

Van de Pol et al. used panoramic radiography of the lower limbs to assess planning and axis correction after high tibial osteotomy. The authors observed that when aiming at a mechanical axis over the lateral tibial spine, a hypocorrection was obtained. However, due to a dynamic valgus displacement obtained during the gait loading phase, a good clinical correction was observed (average valgus $3^{\circ}$ ). The authors concluded that the planning and calculation of the wedge in the preoperative period are essential and, even so, the planned wedge does not always correspond to the wedge used during the intraoperative period for correction. Our study did not evaluate patients in the pre- or postoperative period of tibial osteotomy. In the future we plan to set up a cohort of patients with different characteristics for evaluation through panoramic radiography, such as patients in the postoperative period of tibial or femoral osteotomy, patients with fracture sequelae or patients with congenital deformities. However, it is necessary to emphasize here the importance and validation of panoramic radiography in deformity caused by different reasons and not only osteoarthritis [11].

Gunther et al. assessed the overlapping relationship of the proximal tibia and fibula and determined knee rotation using panoramic radiography. The authors discussed the importance of careful assessment of limb rotation during radiographic studies, and through this work, they established a formula to determine the rotation, in degrees, of the knee. Our study did not evaluate the rotation of the limb on panoramic radiography and we consider this an important limitation of the evaluation. Rotation of the limb is essential in the demarcation of points for measuring the mechanical axis (center of the femoral head, center of the tibial spines and center of the ankle) and small rotational deviations can influence the measurements obtained [22].

Van Raaij et al. studied the correlation between the measurement of the tibiofemoral anatomical angle using simple radiography of the knee and the actual measurement using panoramic radiography. The authors found a high inter-observer correlation in determining the mid-diaphyseal line and a low correlation between the tibiofemoral angle when comparing simple and panoramic radiographs. In conclusion, they pointed out that simple radiographs of the knee cannot replace the panoramic view when an accurate assessment of alignment measures is required [23].

Another theme that has been emerging in the literature is the importance of 
validating image storage and processing systems. With the expansion of applied technologies, it is necessary to establish good parameters of use so as not to lose the accuracy of the desired measures. Some studies in the literature compared the reliability of measurements in filing and communication systems (PACS) and in printed images. Nowicki et al. concluded that the two methods have good reliability and that measures of deformity and discrepancy of limbs can also be performed using the computerized system (PACS). Our study was limited to computerized methods since in our institution there is no conventional printed method [24].

Sled et al. point to constant positioning errors in obtaining panoramic radiographs with a load. The authors emphasize that accurate and reliable measurements of the knee are often unavailable, and this is due to the variability of measurement parameters and radiographic methods. For example, the positioning of the foot, knee rotation and knee flexion can directly affect the measurements found [25].

Babazadeh et al. studied axis deviations by comparing panoramic radiography of the lower limbs with computed tomography and computer-aided navigation. They concluded that the lower limb view is an effective, low-invasive, inexpensive method with less radiation for assessing deformity when compared to the other methods evaluated. $(\mathrm{PMI} \times \mathrm{TC})$ [26].

Bowman et al. assessed the inter-observer and intra-observer reliability of the mechanical axis measurement before and after TKA using panoramic radiography in an Australian population. The authors used examiners with different levels of training and found good correlation and strong reliability regardless of the level of training of the observer [8].

In our view, radiography of the lower limbs can be used to measure the alignment of the mechanical axis with strong reliability between the different evaluators, regardless of experience and degree of training. This information is important for evaluating alignment measures during clinical practice, for assessing the severity of the deformity, for preoperative planning and evaluation of postoperative results. We believe that, over time, panoramic radiography has been neglected in orthopedic daily life due to the mechanization of the evaluation processes and the underestimation of the potential of their information.

\section{Conclusion}

Our study found highly significant intra-observer and inter-observer agreement in determining the mechanical axis of the lower limb through panoramic radiography of the lower limbs in patients undergoing total knee arthroplasty, regardless of the evaluator's experience.

\section{Conflicts of Interest}

The authors declare no conflicts of interest regarding the publication of this paper. 


\section{References}

[1] Cooke, T.D. (2002) Definition of Axial Alignment of the Lower Extremity. The Journal of Bone \& Joint Surgery, 84, 146-147.

[2] Sharma, L., Song, J., Felson, D.T., Cahue, S., Shamiyeh, E. and Dunlop, D.D. (2001) The Role of Knee Alignment in Disease Progression and Functional Decline in Knee Osteoarthritis. JAMA, 286, 188-195. https://doi.org/10.1001/jama.286.2.188

[3] Zhang, Y. and Jordan, J.M. (2008) Epidemiology of Osteoarthritis. Rheumatic Disease Clinics of North America, 34, 515-529.

[4] Werner, F.W., Ayers, D.C., Maletsky, L.P. and Rullkoetter, P.J. (2005) The Effect of Valgus/Varus Malalignment on Load Distribution in Total Knee Replacements. Journal of Biomechanics, 38, 349-355. https://doi.org/10.1016/j.jbiomech.2004.02.024

[5] Sikorski, J.M. (2008) Alignment in Total Knee Replacement. The Journal of Bone and Joint Surgery, 90, 1121-1127. https://doi.org/10.1302/0301-620X.90B9.20793

[6] Berend, M. (2010) Consequences of Malalignment in Total Knee Arthroplasty: Few If Any-Opposes. Seminars in Arthroplasty, 21, 99-101. https://doi.org/10.1053/j.sart.2009.12.009

[7] Turcot, K., Armand, S., Lübbeke, A., Fritschy, D., Hoffmeyer, P. and Suvà, D. (2013) Does Knee Alignment Influence Gait in Patients with Severe Knee Osteoarthritis? Clinical Biomechanics, 28, 34-39. https://doi.org/10.1016/j.clinbiomech.2012.09.004

[8] Bowman, A., Shunmugam, M., Watts, A.R., Bramwell, D.C., Wilson, C. and Krishnan, J. (2016) Inter-Observer and Intra-Observer Reliability of Mechanical Axis Alignment before and after Total Knee Arthroplasty Using Long Leg Radiographs. Knee, 23, 203-208. https://doi.org/10.1016/j.knee.2015.11.013

[9] Abu-Rajab, R.B., Deakin, A.H., Kandasami, M., Mcglynn, J., Picard, F. and Kinninmonth, A.W. (2015) Hip-Knee-Ankle Radiographs Are More Appropriate for Assessment of Post-Operative Mechanical Alignment of Total Knee Arthroplasties than Standard AP Knee Radiographs. Journal of Arthroplasty, 30, 695-700. https://doi.org/10.1016/j.arth.2014.11.024

[10] Lee, S.C., Gondalia, V., Hwang, B.Y., et al. (2016) Is End-Stage Lateral Osteoarthritic Knee Always Valgus? Mechanical Alignment Analysis and Radiographic Severity Assessment. Journal of Orthopaedics and Traumatology, 17, 35-40. https://doi.org/10.1007/s10195-015-0356-9

[11] Van de Pol, G.J., Verdonschot, N. and Van Kampen, A. (2012) The Value of the Intra-Operative Clinical Mechanical Axis Measurement in Open-Wedge Valgus High Tibial Osteotomies. Knee, 19, 933-938. https://doi.org/10.1016/j.knee.2012.02.003

[12] Long, W.J., Bryce, C.D., Hollenbeak, C.S., Benner, R.W. and Scott, W.N. (2014) Total Knee Replacement in Young, Active Patients: Long-Term Follow-Up and Functional Outcome: A Concise Follow-Up of a Previous Report. The Journal of Bone and Joint Surgery, 96, e155. https://doi.org/10.2106/JBJS.M.01259

[13] Lyras, D.N., Greenhow, R. and Loucks, C. (2017) Restoration of the Mechanical Axis in Total Knee Artrhoplasty Using Patient-Matched Technology Cutting Blocks. A Retrospective Study of 132 Cases. The Archives of Bone and Joint Surgery, 5, 283-289.

[14] Shrout, P.E. and Fleiss, J.L. (1979) Intraclass Correlations: Uses in Assessing Rater Reliability. Psychological Bulletin, 86, 420-428. https://doi.org/10.1037/0033-2909.86.2.420

[15] Hunter, D.J. and Wilson, D.R. (2009) Role of Alignment and Biomechanics in Osteoarthritis and Implications for Imaging. Radiologic Clinics of North America, 47, 
553-566. https://doi.org/10.1016/j.rcl.2009.04.006

[16] Hannan, M.T., Felson, D.T. and Pincus, T. (2000) Analysis of the Discordance between Radiographic Changes and Knee Pain in Osteoarthritis of the Knee. The Journal of Rheumatology, 27, 1513-1517.

[17] Skytta, E.T., Haapamaki, V., Koivikko, M., Huhtala, H. and Remes, V. (2011) Reliability of the Hip-Toankle Radiograph in Determining the Knee and Implant Alignment after Total Knee Arthroplasty. Acta Orthopaedica Belgica, 77, 329-335.

[18] Brouwer, G.M., Van Tol, A.W., Bergink, A.P., Belo, J.N., Bernsen, R.M.D., Reijman, M., et al. (2007) Association between Valgus and Varus Alignment and the Development and Progression of Radiographic Osteoarthritis of the Knee. Arthritis \& Rheumatology, 56, 1204-1211. https://doi.org/10.1002/art.22515

[19] Hinman, R., May, R. and Crossley, K. (2006) Is There an Alternative to the Full-Leg Radiograph for Determining Knee Joint Alignment in Osteoarthritis? Arthritis \& Rheumatology, 55, 306-313. https://doi.org/10.1002/art.21836

[20] Kraus, V., Vail, T. and Worrell Mcdaniel, G.A. (2005) Comparative Assessment of Alignment Angle of the Knee by Radiographic and Physical Examination Methods. Arthritis \& Rheumatology, 52, 1730-1735. https://doi.org/10.1002/art.21100

[21] Issa, S., Dunlop, D., Chang, A., Song, J., Prasad, P., Guermazi, A., et al. (2007) FullLimb and Knee Radiography Assessments of Varus-Valgus Alignment and Their Relationship to Osteoarthritis Disease Features by Magnetic Resonance Imaging. Arthritis \& Rheumatology, 57, 398-406. https://doi.org/10.1002/art.22618

[22] Günther, K.P. and Sun, Y. (1999) Reliability of Radiographic Assessment in Hip and Knee Osteoarthritis. Osteoarthritis and Cartilage, 7, 239-246. https://doi.org/10.1053/joca.1998.0152

[23] Van Raaij, T.M., Brouwer, R.W., Reijman, M., Bierma-Zeinstra, S.M. and Verhaar, J.A. (2009) Conventional Knee Films Hamper Accurate Knee Alignment Determination in Patients with Varus Osteoarthritis of the Knee. Knee, 16, 109-111. https://doi.org/10.1016/j.knee.2008.10.003

[24] Nowicki, P.D., Vanderhave, K.L., Farley, F.A., Kuhns, L.R., Dahl, W. and Caird, M.S. (2012) Reliability of Digital Radiographs for Pediatric Lower Extremity Alignment. Journal of Pediatric Orthopaedics, 32, 714-718. https://doi.org/10.1097/BPO.0b013e3182694e07

[25] Sled, E.A., Sheehy, L.M., Felson, D.T., Costigan, P.A., Lam, M. and Cooke, T.D. (2011) Reliability of Lower Limb Alignment Measures Using an Established Landmark-Based Method with a Customized Computer Software Program. Rheumatology International, 31, 71-77. https://doi.org/10.1007/s00296-009-1236-5

[26] Babazadeh, S., Dowsey, M.M., Bingham, R.J., Ek, E.T., Stoney, J.D. and Choong, P.F. (2013) The Long Leg Radiograph Is a Reliable Method of Assessing Alignment When Compared to Computer-Assisted Navigation and Computer Tomography. Knee, 20, 242-249. https://doi.org/10.1016/j.knee.2012.07.009 